\title{
Distribution of Contact Pressures between the Pivoting Support and Tracks for Super Cranes
}

\author{
J. T. Guo, ${ }^{1}$ S. F. Li, ${ }^{2}$ and L. Feng ${ }^{3}$ \\ School of Civil and Safety Engineering, Dalian Jiaotong University, Dalian, China \\ 1 gjtan@djtu.edu.cn \\ 2 lsf_910tonghua@163.com \\ 3 805913500@qq.com
}

The pivoting support is a core structure that assures rotation of super full-rotation cranes. The surface-surface sliding-friction pivoting support is a new potentiality, yet several technical problems still need to be solved, e.g., equilibrium between the pivoting support and tracks. The distribution of contact pressures of three pivoting support models for four typical load cases was calculated. Analysis of contact pressures was based on the finite element method, and the distribution curves for circumferential contact pressures were plotted. The pivoting support design effect on the contact pressures is studied, demonstrating an optimum structure that provides for the contact pressures to be well distributed.

Keywords: full-rotation crane, pivoting support, sliding friction, contact pressure.

Introduction. With the development of the off-shore oil drilling platforms, there is an increasing demand for higher capacity off-shore cranes. Since 1963, many developed countries have invested in research and development of higher capacity off-shore cranes. For instance, Holland refitted oil tankers into crane vessels with a capacity of 150 ton. More recently, the maximum carrying capacity of single-suspension-armed cranes has reached 7000 ton. China has successfully debveloped non-revolving floating cranes $[1,2]$ with load-bearing capacities of 1300 and 1600 ton, and full-rotation-non-self-propelled floating cranes [3] with a load-bearing capacity of 4000 ton. In 2008, a full-rotation-selfpropelled marine crane [4] has successfully accomplished operations, which involved its rotation by 360 degrees under the load of 7500 ton.

The pivoting support is one of the core mechanisms of full-rotation cranes, as it supports the crane and assures its rotation. The pivoting support connects the arm shelf of the crane to the lower section of the crane, bears loads from above the slewing platform of cranes, and passes the loads to tracks to the bearing platform via its bottom. Thus, the form of pivoting support has a dominant influence on the construction of the hoisting devices above the slewing platform, the supporting tube below the slewing platform, and the design of the slewing drive device.

There are two types of structural pivoting support devices; the bogie and the roller [5]. The traditional type of full-circle slewing cranes commonly utilizes the bogie support device with equalizer beams, which are based on equilibrium; therefore, the crane is still supported despite the size, a higher barycenter and a lower stability, when the crane load increases, whereas the multi-support-track-type pivoting supports with no equalizer beams have a compact structure, lower barycenter and better stability. Also, they are simpler to manufacture and install. This type of pivoting support is widely used for large-sized, full-rotation offshore platform cranes. With an increase in the crane load, the crane-load moment increases, and stress in the rollers cause them to become unbalanced and overloaded, which contributes to a lower hosting capacity.

The recent idea for sliding pivoting support implies a slewing mechanism of sliding friction, with chutes instead of rollers and surface-to-surface contact pairs utilized between 
the settled tracks and the chutes set at the bottom of the front half of the pivoting support. This type of support also has a more compact structure, a lower barycenter, a higher stability, a larger bearing surface and a higher loading capacity. However, the pivoting support is still under development, with many technical problems to be solved. That is, the pivoting support mechanism has to bear axial and radial loads, overturning moment, driving torques during rotation, friction between the tracks, and the combination of them all. The loss of balance takes place during the distribution of pressures between the rotary beams and the interface of the tracks, in both circumferential and radial directions. The loss of balance unduced by the contact stresses always leads to the deformation of bearing chutes, interference on the rotation performance of cranes, and causes serious wear and abrasion, which impairs both the carrying capacity and the serving life of cranes.

Based on the literature used for this study, the numerical analysis method [6] is used for analyzing contact stresses of the sliding-friction pivoting support, as well as that of the pivoting supporting of $2 \times 8000$ ton off-shore crane. Within framework of this study, analyses on the distribution of contact pressures of three kinds of pivoting support models under four typical loading conditions are conducted. Curves depicting the relationship between the contact pressures and the circumferential angle are plotted, with values obtained from the edges of the sliding contact surface. The circumferential and radial distributions of contact pressures between pivoting supports and tracks are discussed. The effects of pivoting support on contact pressures are analyzed and a reasonable structuring providing the optimal distribution of contact pressures is proposed.

\section{Methods and Analysis.}

1.1. Computational Model and Loading Conditions. Pivoting support mechanism and Computational Model. The pivoting support structuring, as shown in Fig. 1, consists of upper platform girders, supporting columns, and lower girders. There is a pair of hinge lugs (on hinge spot $A$, in Fig. 1) on the rear spars of the platform girders, and 2 pairs of hinge lugs on the upper front beam (on hinge spot $B$ and $C$, in Fig. 1), which respectively join the A-frame and boom with a hinge allowing a rotation at any angle in the amplitude plane. The girder on the bottom of the pivoting support is subdivided into two parts. The front half that contacts the surface of the tracks is called a rotary beam. A box structure on the bottom bearing surface has two concentric chutes that correspond to the tracks on the bearing platform, providing a sliding contact with the tracks. The rear half has the equalizer beams, while for the two concentric chutes on the bearing platform a rolling wheel rack is used to balance the overturning moment, which allows one to avoid capsizing, ultimately achieving a full-circle rotation of the whole crane.

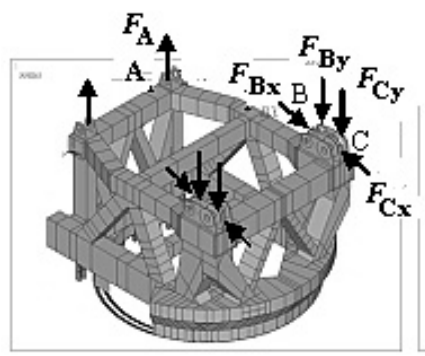

a

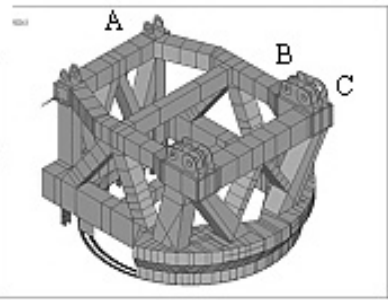

b

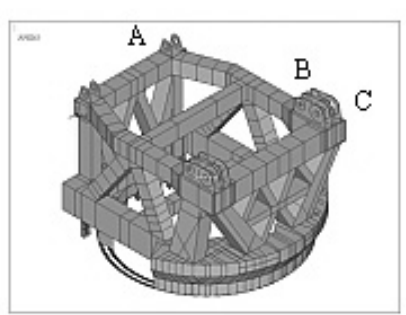

$\mathrm{c}$

Fig. 1. Models of sliding-friction pivoting support: (a) model I; (b) model II; (c) model III.

The supporting columns contact the platform girders, leaving the lower girders with a sufficient room for pivoting support, and upwards pass loading. This study is dedicated to three different kinds of supporting column structuring models, as shown in Fig. 1. Edge supporting columns in model I are straighter and shorter in distance between the columns, 
while the edge supporting columns in model II are leaner and wider between the columns. Although distances in model III are the same with that of model II, there are additional secondary girders in model III that form a pattern of a double-decker $W$.

Sliding-friction pivoting support is a box structure formed by welded plates, which is sumulated by shell elements in the proposed finite element model. These elements elements are placed with consideration of their physical locations and assigned the attributes corresponding to the physical thickness of the simulated plates. The secondary part, a non-load-bearing structure, uses mass elements to simulate the effect of the counterweight. The tracks, which are settled on an off-shore platform, have a comparatively higher rigidity and small deformation, and thus are reduced in the analysis to rigid bodies. However, the rotary beam and the sliding surface are considered as elastic bodies, which make up a rigid-flexible contact pair, in order to take into account their deformations. Several 3D surface-to-surface contact elements are used to simulate the contact between the tracks and the sliding surfaces of rotary beams.

1.2. Load Cases. At the primary designing stage, the rotation condition was not considered yet, so only four typical load cases were analyzed, according to the lifting performance curve of the full-rotation cranes of 8000 ton.

Load case 1 - the lower limit of working range of the full load (the hoisting capacity), when pressure in the front beam hinge point (hinge spot $C$ in Fig. 1) reaches its maximum value.

Load case 2 - the hoisting load and the working range of the jib attain a certain level, while pressure in the A-frame hinge point (hinge spot $B$ in Fig. 1) reaches its maximum.

Load case 3 - the hoisting capacity, where the main jib has the maximum working range while running.

Load case 4 - the limit of the range that the main jib reaches without any load.

The load case with a hoisting capacity of 8000 ton and a working range of $50 \mathrm{~m}$ was considered as referenced. When calculating different load cases, according to the position of the jib of the crane, the external forces were assumed to be applied in every hinge point of the girders on the upper platform: $F_{A}$ in point $A, F_{B y}$ and $F_{B x}$ in point $B, F_{C x}$ and $F_{C y}$ in point $C$ (as shown in Fig. 1a). These forces were treated as concentrated loads applied to the corresponding nodes of the finite element model.

Rigid-flexible contact parameters were assigned, in accordance with the research literature [7-11]. Study on the contact relationship between the bearing chutes of slidingfriction pivoting support devices and the platform tracks was performed by the finite element method (FEM).

\section{Results.}

2.1. Pivoting Support and Distribution of Contact Stresses in Tracks. Contact pressures between the bearing chutes and the platform tracks were calculated using FEM. The distribution of contact pressure between the pivoting support and the tracks is plotted in Fig. 2. It can be seen that there are differences between the contact pressures in the two chutes, and there exists a loss of balance among contact pressures. The loss of balance is proved by different squeezing levels between the sliding surfaces of the rotary beam and the tracks, as well as by the formation of contact spots. In the contact spot areas, contact pressures are high, whereas outside those areas they are lower. The tracks are arc-shaped, and only part of them bear pressures within $120-130^{\circ}$, where they overlap with the rotary beam of the front half of the pivoting support, while the pressures are lower or close to zero in the other parts of the tracks. Thus, it is expedient to focus only on the overlapped areas, when analyzing the contact pressures.

For simplicity, the contact pressures for the two edges of the outer chute and two edges of the inner chute were obtained, and the pressure-angle distribution curves of each edge were constructed. The edges are numbered from outer to inner ones, with the outer edge of the outer chute as edge I, the inner edge of the outer chute as edge III, the outer 


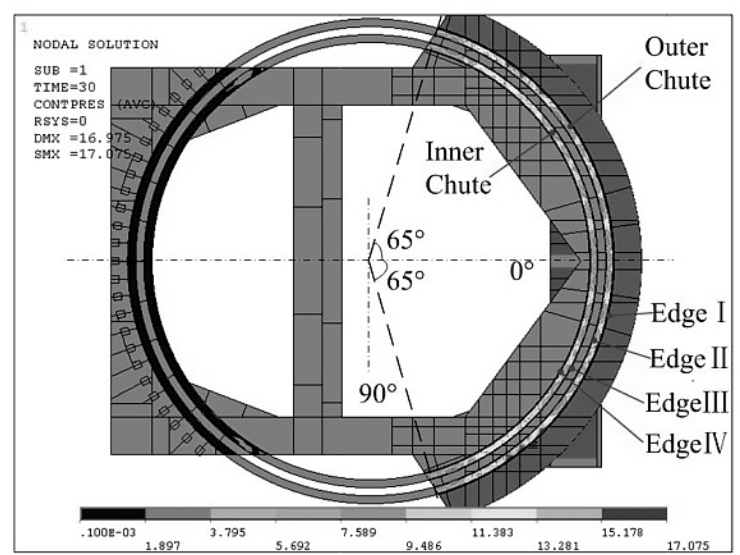

Fig. 2. Diagram of contact pressures between pivoting support and tracks.

edge of the inner chute as edge III, and the inner edge of the inner chute as edge IV, as shown in Fig. 2.

Insofar as the pivoting support is a symmetrical structure, there always exists a symmetrical surface under lifting condition if the rotation condition is not taken into consideration. The stresses and deformations on pivoting support two sides are equal, as well as the contact status of the tracks. Therefore it is observed from only one side of the track, which is within $0-65^{\circ}$. A distribution curve of circumferential pressures on the contact surfaces was constructed using their values at the inner and outer edges of the three models under different loadings. Distribution curves of contact pressures of model I under load case 1 are presented in Fig. 3. Distributions of other models and load cases, as they have quite similar characteristics as those of model I, are omitted for brevity. Figure 4 shows contact pressure-angle curves of the edge of the outer chute (edge I) for three kinds of models.

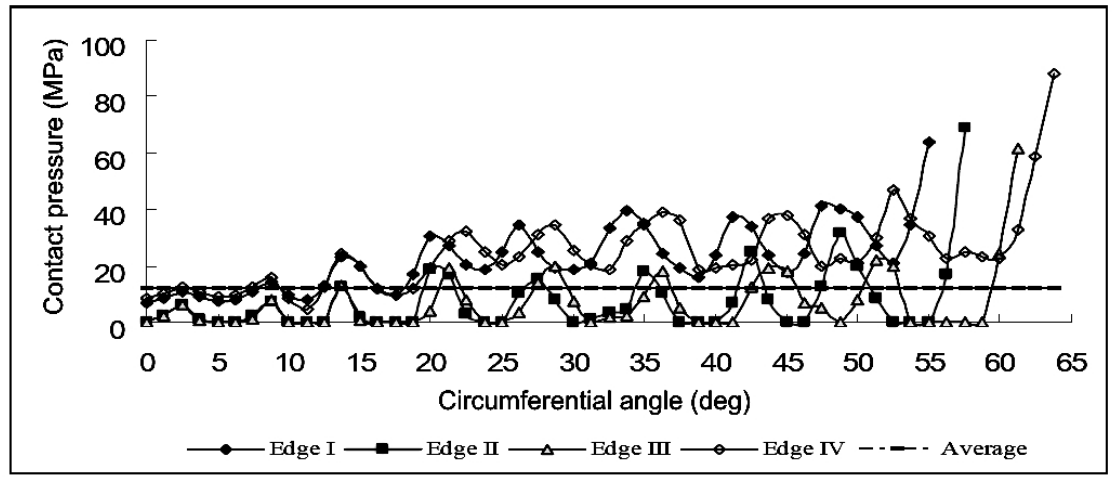

Fig. 3. Contact pressure-angle curves of the edges, model I under load case 1.

Figures 3 and 4 demonstrate that the contact pressure values of the outer edge of the outer chute, as well as of the inner edge of the inner chute, are generally higher than those of the track surface. The contact pressures are distributed in a wavy pattern, where pressures reach their minimum value in the symmetry center of the pivoting support rotary beam, while pressures on both ends reach their maximum. In model I, pressures manifest stong fluctuations with the maximum values at the ends, yet model II provides nearly the same distribution as model III. 


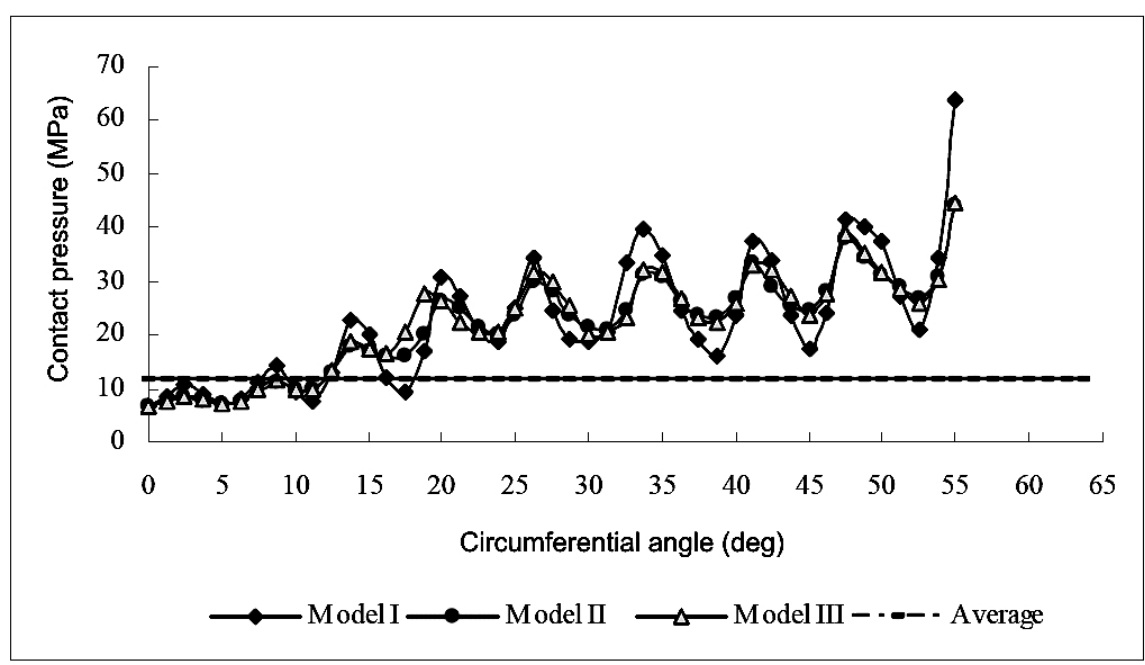

Fig. 4. Contact pressure-angle curves of edge I of three models for load case 1.

2.2. Discussion. The contact pressures induced by the load that emerges when the crane is running, appeared to be lower at the ends but higher in the middle, acting in hinge spots $B$ and $C$ as centered forces (Fig. 4). The pressure then moves downwards along the supporting columns, finally resulting in heavy loads at both ends of the rotary beam. In model I, distances between the supporting columns were shorter, with the maximum distance being at the ends of the beam and the minimum distance in the center of the beam under the loads passing upwards to the rotary beam. In models II and III, distances between the two main supporting points were longer, deviation load on the rotary beam shrunk, and contact pressures gradually smoothed. Additional secondary beams were added in model III. A high and well-distributed rigidity of the beams made it possible to balance the contact pressures, but did not provide any further applications regarding more complex structures.

The distribution of the contact pressures along the rotary beam is controlled by its design features: it is a box structure consisting of welded plates, circumferential risers at the both sides with a higher rigidity, as compared to that of the cavity of the inner part, which causes high contact pressures at the outer edge (Fig. 3, edge I) and the inner edge of the inner chute (edge IV). Between lateral and medial side plates of the rotary beam there are diaphragm plates, while the distances between the diaphragm plates coincide with the wave period (Fig. 4).

Conclusions. The calculation results obtained for three models and 4 load cases show a regular pattern of distribution of contact pressures between the sliding surfaces of the rotary beam and the tracks. The areas, where the maximum contact pressures occurred, were found at the ends of the pivoting support rotary beam. By increasing the distances between the front supporting columns of the pivoting support, the scatter can be reduced, and the difference in contact pressures will decrease. In the rotary beam, contact pressures at the outer edge of the outer chute, as well as at the inner edge of the inner chute, are generally higher than those at the sliding surface. The latter stresses have a wavy distribution pattern with the peaks corresponding to the positions of diaphragm plates and troughs corresponding to the cavity position. The key to improving the equilibrium characteristics of contact pressures between sliding surfaces of the pivoting support rotary beam and the tracks is a rational arrangement of diaphragms of the rotary beam, and settlement on the consistent match of rigidity of the rotary beam. 
1. Y. Shen and Y. M. Wang, "Analysis of large un-rotational floating crane's structure form," Chin. J. Constr. Machinery, No. 3, 439-444 (2005).

2. Y. Shen and Y. M. Wang, "1300t floating crane metal structure finite element analysis," Hoist. Conv. Machinery, No. 4, 4-7 (2005).

3. W. H. Yan and Z. Gui, "The analysis of load-bearing and optimization of rollerdesign on supporting structure of 4000t floating derrick," J. Mach. Design, No. 8, 122-124 (2007).

4. H. D. Zhang, Y. P. He, and H. L. Li, "Optimization of ballast tanks for 7500t floating crane," Chin. J. Ship Res., No. 2, 49-54 (2010).

5. Y. M. Wang, Y. L. Wang, and M. H. Tong, "Enabling technologies for new rotary support device of ultra-large-scale rotary floating cranes," Chin. J. Mech. Eng., No. 2, 180-184 (2009).

6. J. T. Guo and S. F. Li, "The research of the pivoting support force balance of the super large crane," Adv. Mater. Res., 671-674, 888-892 (2013).

7. J. I. Amasorrain and X. Sagartzazu, "Load distribution in a four contact-point pivoting support," Mech. Mach. Theory, 38, 479-496 (2003).

8. J. Piotrowski and W. Kik, "A simplified model of wheel/rail contact mechanics for non-Hertzian problems and its application in rail vehicle dynamic simulations," Vehicle Syst. Dynamics, 46, 27-48 (2008).

9. W. Sun, W. Qin, and B. Zhu, "Contact analysis of rotation bearing finite element method," Mech. Transmission, No. 6, 73-75 (2010).

10. T. Telliskivi and U. Olofsson, "Contact mechanics analysis of measured wheel-rail profiles using the finite element method," Proc. Inst. Mech. Eng., Part F, J. Rail Rapid Transit, 215, 65-72 (2001).

11. S. Satyanarayana and S. N. Melkote, "Finite element modeling of fixture-work piece contacts: single contact modeling and experimental verification," Int. J. Mach. Tools Manufact., 44, No. 9, 903-913 (2004). 